\title{
RATIOS OF REGULATORS IN EXTENSIONS OF NUMBER FIELDS
}

\author{
ANTONE COSTA AND EDUARDO FRIEDMAN
}

(Communicated by William W. Adams)

\begin{abstract}
Let $L / K$ be an extension of number fields. Then

$$
\left.\operatorname{Reg}(L) / \operatorname{Reg}(K)>c_{[L}: \mathbf{Q}\right]\left(\log \left|D_{L}\right|\right)^{m},
$$

where Reg denotes the regulator, $D_{L}$ is the absolute discriminant of $L$, and $c_{[L: O]}>0$ depends only on the degree of $L$. The nonnegative integer $m=$ $m(L / K)$ is positive if $L / K$ does not belong to certain precisely defined infinite families of extensions, analogous to CM fields, along which $\operatorname{Reg}(L) / \operatorname{Reg}(K)$ is constant. This generalizes some inequalities due to Remak and Silverman, who assumed that $K$ is the rational field $\mathbf{Q}$, and modifies those of Berge-Martinet, who dealt with a general extension $L / K$ but used its relative discriminant where we use the absolute one.
\end{abstract}

\section{INTRODUCTION}

Remak [R1] laid down the principle that a number field ought to have a large regulator if and only if it has a large discriminant. In one direction this follows from work of Landau [L, Sie], who proved that $\sqrt{\left|D_{L}\right|}\left(\log \left|D_{L}\right|\right)^{[L: Q]-1}$ is an upper bound for $\operatorname{Reg}(L)$. To obtain an inequality in the opposite sense, Remak considered the field $\mathbf{Q}\left(E_{L}\right)$ generated by the units $E_{L}$ of $L$. The geometry of numbers tells us that $\mathbf{Q}\left(E_{L}\right)$ can be generated by integral elements (units) whose size at every embedding is bounded in terms of $\operatorname{Reg}(L)$. It follows that $\left|D_{\mathbf{Q}\left(E_{L}\right)}\right|$ can be bounded above by a function of $\operatorname{Reg}(L)$. Remak then observed that $\mathbf{Q}\left(E_{L}\right)=L$ unless $L$ is a CM field (a totally imaginary quadratic extension of a totally real field). Thus he proved [R1]

$$
\operatorname{Reg}(L)>C_{N} \log \left(\frac{\left|D_{L}\right|}{N^{N}}\right),
$$

where $L$ is assumed non-CM, $N=[L: \mathbf{Q}]$, and $C_{N}>0$ depends explicitly on $N$. In 1984 Silverman [Sil] improved the dependence on $\log \left|D_{L}\right|$ in (1.1) to

$$
\operatorname{Reg}(L)>2^{-4 N^{2}}\left(\log \left(\frac{\left|D_{L}\right|}{N^{N^{\log _{2}(8 N)}}}\right)\right)^{r_{L}-\rho},
$$

Received by the editors September 25, 1991 and, in revised form, February 18, 1992. 1991 Mathematics Subject Classification. Primary 11R27, 11 R29.

Key words and phrases. Regulator, discriminant, unit-weak extensions.

The first author was partially supported by NSA grant MDA90-H-1019.

Research was supported by the Max Planck Institut für Mathematik and the NSF. 
where $\left|D_{L}\right|>N^{N^{\log _{2}(8 N)}}$ is assumed, $r_{L}$ is the unit rank of $L$, and $\rho=$ $\max _{F} \subset_{L}\left\{r_{F}\right\}$.

It follows from (1.1) that given an integer $N$ and a real number $y$ there are only finitely many non-CM number fields $L$ such that $[L: \mathbf{Q}] \leq N$ and $\operatorname{Reg}(L)<y$. CM fields must be excluded since the regulator, being essentially that of a proper subfield, can have the same value for infinitely many CM fields. We can, however, drop all restrictions on the degree $[L: \mathbf{Q}]$ by using Zimmert's [Z] bound

$$
\operatorname{Reg}(L)>(0.04) 1.05^{[L: Q]} .
$$

In the late 1980s Bergé and Martinet [BM1, BM2] generalized Remak and Silverman's method to the relative case. Given an extension $L / K$ of number fields their idea was to equate the ratio of regulators $\operatorname{Reg}(L) / \operatorname{Reg}(K)$ with the covolume of a lattice produced from the units of $L$. In their approach the absolute norm $\mathrm{N}\left(\mathscr{D}_{L / K}\right)$ of the relative discriminant of $L / K$ appeared naturally and they were able to bound $\operatorname{Reg}(L) / \operatorname{Reg}(K)$ from below by a power of $\log \left(\mathrm{N}\left(\mathscr{E}_{L / K}\right)\right)$.

While Bergé and Martinet's results can be used quite effectively [BM3] if $\mathrm{N}\left(\mathscr{D}_{L / K}\right)$ is large, they are otherwise not so strong. This makes it difficult to obtain inequalities in which $K$ is allowed to vary, say only fixing $[L: \mathbf{Q}$, as there will be in general infinitely many $L / K$ with $\mathrm{N}\left(\mathscr{D}_{L / K}\right)=1$. Our results for totally real fields $[C F]$ suggest that this problem could be overcome by modifying Bergé and Martinet's lattice. We use the lattice associated to the relative units $E_{L / K}$. By definition, $E_{L / K}$ consists of those units of $L$ whose norm to $K$ is a root of unity. Since the covolume of $E_{L / K}$ under the logarithmic embedding is readily related to $\operatorname{Reg}(L) / \operatorname{Reg}(K)$, we can apply Remak's geometric method to bound the absolute discriminant of $\mathbf{Q}\left(E_{L / K}\right)$ from above in terms of $\operatorname{Reg}(L) / \operatorname{Reg}(K)$. It turns out that $\mathbf{Q}\left(E_{L / K}\right)=L$, except when one of the following three conditions holds:

(i) $L=K$.

(ii) The field $L$ is CM (and $K$ is any subfield of $L$ ).

(iii) There is a $\mathrm{CM}$ field $M$ with maximal totally real subfield $k$ such that $K$ is a quadratic extension of $k, K \neq M$, and $L=M K$.

We call the extension $L / K$ unit-weak if it satisfies (i), (ii) or (iii) above.

Theorem. Let $E_{L / K}$ denote, as above, the relative units of an extension $L / K$ of number fields. Assume that $\left|D_{L}\right|>3 N^{N}$, where $D_{L}$ is the discriminant of $L / \mathbf{Q}$ and $N=[L: \mathbf{Q}]$. Then

$$
\frac{\operatorname{Reg}(L)}{\operatorname{Reg}(K)}>\frac{C}{N^{2 r}}\left(\log \left(\frac{\left|D_{L}\right|}{N^{N}}\right)\right)^{m},
$$

where $r=\operatorname{rank}\left(E_{L / K}\right)=r_{L}-r_{K}$ is the difference of the unit ranks of $L$ and $K$, Reg is the regulator, and $C>0$ is a computable absolute constant. The nonnegative integer $m$ is positive if $L / K$ is not unit-weak (see the above definition). In general, $m=m(L / K)=r-\max _{F \varsubsetneqq L}\left\{\operatorname{rank}\left(E_{L / K} \cap F\right)\right\}$, where the maximum is taken as $F$ runs over all proper subfields of $L$.

We actually prove the somewhat stronger inequality (3.7) in which $\operatorname{Reg}(L) / \operatorname{Reg}(K)$ is replaced by the regulator of $E_{L / K}$. The exponent $m$ of $\log \left(\left|D_{L}\right|\right)$ in (1.2) is likely to be best possible. In any case, $m$ can be computed 
by easy linear algebra, without knowledge of any unit, as long as one knows all the subfields of $L$ (see the end of $\S 3$ ). In contrast, we do not calculate $C$ here, as we do not obtain a good value. Our proof does yield that one can take $C=1$ and $m=r$, provided we assume that every proper subfield of $L$ is actually a subfield of $K$.

When $L / K$ is unit-weak $m$ vanishes and (1.2) becomes almost useless, however, in this case the ratio of regulators $\operatorname{Reg}(L) / \operatorname{Reg}(K)$ is essentially that of a proper subextension. Unit-weak extensions can thus be treated inductively and represent no essential complication to the problem of bounding $\operatorname{Reg}(L) / \operatorname{Reg}(K)$ from below. We treat unit-weak extensions briefly at the end of $\S \S 2$ and 3 .

A consequence of (1.2) is

Corollary. Given an integer $N$ and a real number $y$, there are at most finitely many extensions $L / K$ such that $[L: Q] \leq N, \operatorname{Reg}(L) / \operatorname{Reg}(K)<y$, and $L / K$ is not unit-weak.

If $L$ is totally real even more is true: Given any real number $y$ there are finitely many pairs of totally real fields $L$ and $K$, with $K \varsubsetneqq L$, such that $\operatorname{Reg}(L) / \operatorname{Reg}(K)<y[C F]$. We do not know yet if this extends to all non-unitweak $L / K$, totally real or not.

\section{THE FIELD GENERATED BY THE RELATIVE UNITS}

Recall that the group of relative units $E_{L / K}$ of an extension $L / K$ of number fields is defined by

$$
E_{L / K}=\left\{\alpha \in E_{L} \mid \operatorname{Norm}_{L / K}(\alpha) \in W_{K}\right\},
$$

where $E_{L}$ denotes the units of $L$ and $W_{K}$ the torsion subgroup of $E_{K}$. The (free) rank of $E_{L / K}$ is $r=r_{L / K}=r_{L}-r_{K}$, where $r_{L}$ is the rank of $E_{L}$. Let $\mathscr{S}_{L}$ denote the set of embeddings of $L$ into $\mathbf{C}$. We embed $E_{L} / W_{L}$ into $\mathbf{R}^{\mathscr{S}_{L}}$ by the map $\mathscr{L}=\mathscr{L}_{L}: E_{L} \rightarrow \mathbf{R}^{\mathscr{S}_{L}}$ defined by

$$
\left(\mathscr{L}_{L}(\alpha)\right)_{\sigma}=(\mathscr{L}(\alpha))_{\sigma}=\log |\sigma(\alpha)|, \quad \sigma \in \mathscr{S}_{L} .
$$

We endow $\mathbf{R}^{\mathscr{S}_{L}}$ with the Euclidean inner product

$$
\left\langle\left(x_{\sigma}\right),\left(y_{\sigma}\right)\right\rangle=\sum_{\sigma \in \mathscr{S}_{L}} x_{\sigma} y_{\sigma} .
$$

Then $\mathscr{L}_{L}\left(E_{L / K}\right)$ is perpendicular to $\mathscr{L}_{L}\left(E_{K}\right)$. A dimension count shows that the Q-spans $\mathbf{Q} \mathscr{L}_{L}\left(E_{L / K}\right)$ and $\mathbf{Q} \mathscr{L}_{L}\left(E_{K}\right)$ of these two lattices are orthogonal complements of each other inside $\mathbf{Q} \mathscr{L}\left(E_{L}\right)$.

Our first goal is to characterize the extensions $L / K$ for which $\mathbf{Q}\left(E_{L / K}\right)$ is a proper subfield of $L$. Slightly more generally, we prove

Proposition 1. Let $L / K$ be an extension of number fields and let $E_{L / K}$ be its group of relative units. Let $E$ be a subgroup of finite index in $E_{L / K}$ and suppose that $E$ is contained in a proper subfield of $L$. Then at least one of (i), (ii), or (iii) below holds:

(i) $L=K$.

(ii) $L$ is $C M$ (and $K \subset L$ is arbitrary).

(iii) There is a $C M$ field $M$ with maximal totally real subfield $k$ such that $K$ is a quadratic extension of $k, K \neq M$, and $L=M K$. 
Conversely, if (iii), (ii), or (i) holds (with $L \neq \mathbf{Q}$ ), then $E_{L / K}$ contains a subgroup $E$ as above.

Proof. The last statement is obvious in cases (i) and (ii). If (iii) holds, let $H \neq K, H \neq M$ be the third field lying strictly between $k$ and $L$. A short computation shows that $E:=E_{H / k} \subset H$ has the same rank as $E_{L / K}$ and $E_{H / k} \subset E_{L / K}$, proving the converse claim.

We now prove the first part of the proposition. Given a subfield $F \subset L$ and an archimedean place $\omega$ of $L$, let $e_{F}(\omega)=e_{L / F}(\omega)=2$ if $\omega$ ramifies in $L / F$; otherwise, let $e_{F}(\omega)=1$. Let $\infty_{F}$ denote the set of archimedean places of $F$. Then

$$
r_{F}+1=\frac{1}{[L: F]} \sum_{\omega \in \infty_{L}} e_{F}(\omega)
$$

because

$$
r_{F}+1=\sum_{\nu \in \infty_{F}} 1=\sum_{\nu \in \infty_{F}} \frac{1}{[L: F]} \sum_{\substack{\omega \in \infty_{L} \\ \omega \mid \nu}} e_{F}(\omega)=\frac{1}{[L: F]} \sum_{\omega \in \infty_{L}} e_{F}(\omega) .
$$

Let $H=\mathbf{Q}(E)$. Then $H \varsubsetneqq L$, by assumption. Since $E \subset E_{H}$, we have $r_{H} \geq r_{L / K}=r_{L}-r_{K}$. From this and (2.3) we obtain

$$
\frac{1}{[L: H]} \sum_{\omega \in \infty_{L}} e_{H}(\omega)+\frac{1}{[L: K]} \sum_{\omega \in \infty_{L}} e_{K}(\omega)>\sum_{\omega \in \infty_{L}} 1 \text {. }
$$

The compositum $H K \subset L$ contains $E$ and $E_{K}$. Modulo torsion, these are disjoint (perpendicular!) subgroups of $E_{L} / W_{L}$ of rank $r_{L}-r_{K}$ and $r_{K}$; hence, the units of $H K$ have rank $r_{L}$. If $H K \neq L$, then $L$ must be a CM field, in which case the proof is done. We may therefore assume $H K=L$. Then we cannot simultaneously have $e_{H}(\omega)=2$ and $e_{K}(\omega)=2$ for $\omega \in \infty_{L}$. Hence,

$$
\left(\frac{1}{[L: H]}+\frac{1}{[L: K]}\right) \sum_{\omega \in \infty_{L}} 1+\max \left(\frac{1}{[L: H]}, \frac{1}{[L: K]}\right) \sum_{\omega \in \infty_{L}} 1>\sum_{\omega \in \infty_{L}} 1 .
$$

By assumption, $[L: H] \geq 2$. Thus, either $[L: H]=2$ or $[L: K]=2$ (we dismiss the trivial case $L=K$ ).

We first assume $[L: K]=2$. Let $\tau$ be the nontrivial element of $\operatorname{Gal}(L / K) \cong$ $\mathbf{Z} / 2 \mathbf{Z}$. For $\alpha \in E \subset E_{L / K}$, we have $\operatorname{Norm}_{L / K}(\alpha) \in W_{K}$; therefore, $\tau(\alpha)=$ $\eta \alpha^{-1}, \eta \in W_{K}$. By passing, as we may, to a subgroup of finite index in $E$, we can assume $\tau(\alpha)=\alpha^{-1}$; hence, $\tau$ induces a nontrivial field automorphism of $H=\mathbf{Q}(E)$. Let $H_{\tau}$ be its fixed field so that $\left[H: H_{\tau}\right]=2$. Since $H_{\tau} \subset L_{\tau}=K$, we must have either $H \cap K=H_{\tau}$ or $H \cap K=H$. In the latter case we would have $E \subset K$. But then $E \subset K \cap E_{L / K}=W_{K}$. Since $E$ has finite index in $E_{L / K}$, this could only happen if $L$ is CM. We may thus assume $H \cap K=H_{\tau}$. Then $E \subset H \cap E_{L / K}=E_{H / H \cap K} \subset E_{L / K}$. Since $E$ has finite index in $E_{L / K}$, $r_{H / H \cap K}=r_{L / K}$. From this and (2.3) we find

$$
\frac{1}{[L: H]} \sum_{\omega \in \infty_{L}} e_{H}(\omega)-\frac{1}{[L: H \cap K]} \sum_{\omega \in \infty_{L}} e_{H \cap K}(\omega)=\sum_{\omega \in \infty_{L}} 1-\frac{1}{2} \sum_{\omega \in \infty_{L}} e_{K}(\omega) .
$$

Since $[L: H \cap K]=2[L: H]$, we have

$$
\frac{1}{[L: H]} \sum_{\omega \in \infty_{L}}\left(2 e_{H}(\omega)-e_{H \cap K}(\omega)\right)=\sum_{\omega \in \infty_{L}}\left(2-e_{K}(\omega)\right) .
$$


Observe that if $\omega$ ramifies in $L / K$, then $\omega$ ramifies in $L / H \cap K$ but not in $L / H$ (since $L=H K$ ). Thus, if $e_{K}(\omega)=2$, then $2 e_{H}(\omega)-e_{H \cap K}(\omega)=0$. If $e_{K}(\omega)=1$, then $2 e_{H}(\omega)-e_{H \cap K}(\omega) \leq 2$. It now follows from (2.5) that $[L: H]=2$ and that $e_{H}(\omega)=2$ if and only if $e_{K}(\omega)=1$. Hence $[L: H]=$ $2=[H: H \cap K]=[K: H \cap K]$ and all archimedean places of $L$ ramify in either $L / K$ or $L / H$, but none in both extensions. It follows that $L / K$ satisfies condition (iii) in the proposition (let $k=K \cap H$ and let $M \neq K, M \neq H$, be the third field lying strictly between $k$ and $L$ ). This proves Proposition 1 when $[L: K]=2$.

If $[L: K]>2$, then (2.4) implies $[L: H]=2$. The strategy now is to reverse the roles of $H$ and $K$ and thereby reduce the proof to the quadratic case that we just handled. Recall that if $F$ is any subfield of $L$, then the $Q$ spans of $\mathscr{L}\left(E_{L / F}\right)$ and $\mathscr{L}\left(E_{F}\right)$ are orthogonal with respect to the (R-valued) inner product (2.2). By construction, $\mathscr{L}(E) \subset \mathscr{L}\left(E_{H}\right)$. Since $E$ has finite index in $E_{L / K}, \mathbf{Q} \mathscr{L}(E)=\mathbf{Q} \mathscr{L}\left(E_{L / K}\right)$. Hence

$$
\mathbf{Q} \mathscr{L}\left(E_{L / H}\right)=\mathbf{Q} \mathscr{L}\left(E_{H}\right)^{\perp} \subset \mathbf{Q} \mathscr{L}\left(E_{L / K}\right)^{\perp}=\mathbf{Q} \mathscr{L}\left(E_{K}\right),
$$

where ${ }^{\perp}$ denotes the orthogonal complement inside $\mathbf{Q} \mathscr{L}\left(E_{L}\right)$. Since the kernel $W_{L}$ of $\mathscr{L}$ is finite, (2.6) shows that $E_{L / H}^{n} \subset E_{K}$ for some positive integer $n$. Thus $E^{\prime}:=E_{L / H}^{n}$ has finite index in $E_{L / H},[L: H]=2$, and $\mathbf{Q}\left(E^{\prime}\right) \subset K$, a proper subfield of $L$; but this is the quadratic case of the proposition, so the proof is done.

We conclude this section with a brief discussion of the unit-index $u_{L / K}$ of a unit-weak extension $L / K$. We assume first that $K \neq L$ and that $L$ is not CM. Let $k$ and $M$ be as in (iii) above. Denote by $K$ and $H$ the two remaining fields lying strictly between $k$ and $L$. Let $\tau_{H}, \tau_{K}$, and $\tau_{M}=\tau_{H} \tau_{K}$ be the nontrivial automorphisms of $L / H, L / K$, and $L / M$. Since we assume that $L$ is not $\mathrm{CM}$, at least one archimedean place of $k$ ramifies in $H$; hence, at least one archimedean place of $K$ ramifies in $L$. Thus $W_{K}=\{ \pm 1\}$ and -1 is not a norm in $L / K$, whence $\operatorname{Norm}_{L / K}\left(E_{L / K}\right)=\{+1\}$. Equivalently, $\tau_{K}(\alpha)=\alpha^{-1}$ for $\alpha \in E_{L / K}$. Hence, $\operatorname{Norm}_{L / M}(\alpha)=\alpha \tau_{H}\left(\tau_{K}(\alpha)\right)=\alpha / \tau_{H}(\alpha)$. Therefore, $\operatorname{Norm}_{L / M}(\alpha)=1$ if and only if $\alpha \in E_{L / K} \cap H=E_{H / k}$. In short, Norm $_{L / M}$ induces an injection of $E_{L / K} / E_{H / k}$ into $W_{M}=E_{M / k}$. As $W_{M}^{2}=$ $\operatorname{Norm}_{L / M}\left(W_{m}\right) \subset \operatorname{Norm}_{L / M}\left(W_{L}\right)$ and $W_{M}$ is cyclic, we have $u_{L / K}:=\left[E_{L / K}\right.$ : $\left.W_{L} E_{H / k}\right]=1$ or 2 .

So far we have assumed that $L$ is not CM. If $L$ is CM, let $H$ be its maximal totally real subfield. It is well known that $\left[E_{L}: W_{L} E_{H}\right]=1$ or 2 [R2]. It follows that $u_{L / K}:=\left[E_{L / K}: W_{L} E_{H / k}\right]=1$ or 2 , where $k=H \cap K$. Finally, if $L=K$ we let $H=k=\mathbf{Q}$ and $u_{L / K}=1$.

We have thus defined, whenever $L / K$ is unit-weak, a subextension $H / k$ and a unit-index $u_{L / K}:=\left[E_{L / K}: W_{L} E_{H / k}\right]=1$ or 2 . When $L$ is $\mathrm{CM}$ and $K=\mathbf{Q}, u_{L / Q}$ is just the usual unit-index of $L$. In the next section we relate the regulators of $E_{L / K}$ and $E_{H / k}$ using $u_{L / K}$. Notice that $H / k$ itself is not unit-weak unless $r_{L / K}=0$.

\section{Proof of Theorem}

We begin with the definition of the regulator of relative units $\operatorname{Reg}\left(E_{L / K}\right)$. Pick $\alpha_{1}, \alpha_{2}, \ldots, \alpha_{r}$ to be independent generators of $E_{L / K} / W_{L}$, the relative 
units modulo torsion. Let $M$ be the matrix $M=\left(\log \left\|\alpha_{i}\right\|_{\omega}\right)$, where $1 \leq i \leq r$, $\omega$ runs over the set $\infty_{L}$ of archimedean places of $L$, and \|\|$_{\omega}$ denotes the normalized absolute value at $\omega$ (so that \|\|$_{\omega}=||_{\omega}^{2}$ if $\omega$ is complex, and \|\|$_{\omega}=\|\left.\right|_{\omega}$ otherwise). For each place $\nu \in \infty_{k}$, fix a place $\omega_{\nu} \in \infty_{L}$ lying above $\nu$. Then $\operatorname{Reg}\left(E_{L / K}\right)$ is the absolute value of the determinant of the submatrix of $M$, which results when we delete from $M$ the rows corresponding to the $\omega_{\nu}$ 's. In [CF, Theorem 1] we showed, for $L / K$ of any signature,

$$
\operatorname{Reg}\left(E_{L / K}\right)=\frac{1}{\left[E_{K}: W_{K} \operatorname{Norm}_{L / K}\left(E_{L}\right)\right]} \frac{\operatorname{Reg}(L)}{\operatorname{Reg}(K)} .
$$

We also related [CF, Lemma 2.1] $\operatorname{Reg}\left(E_{L / K}\right)$ to the $r$-dimensional volume $V_{L}\left(E_{L / K}\right)$ of a fundamental domain for $\mathscr{L}\left(E_{L / K}\right)($ see $(2.1))$,

$$
V_{L}\left(E_{L / K}\right)=[L: K]^{\left(r_{1}(K)+r_{2}(K)\right) / 2} 2^{\left(r_{2}(K)-r_{2}(L)\right) / 2} \operatorname{Reg}\left(E_{L / K}\right),
$$

where $\left(r_{1}, r_{2}\right)$ denotes the number of (real, complex) places. The Euclidean structure (which normalizes volume) is given by $\left\|\left(x_{\sigma}\right)\right\|^{2}=\left\langle\left(x_{\sigma}\right),\left(x_{\sigma}\right)\right\rangle$, as in (2.2). For $\alpha \in E_{L}$ we write $\|\alpha\|$ instead of $\|\mathscr{L}(\alpha)\|$. Thus,

$$
\|\alpha\|^{2}:=\sum_{\sigma \in \mathscr{S}_{L}}(\log |\sigma(\alpha)|)^{2},
$$

where $\mathscr{S}_{L}$ denotes the set of all embeddings of $L$ into $\mathrm{C}$. We will need the lower bound [F, (3.21)]

$$
\|\alpha\|>\frac{C^{\prime}}{\sqrt{N}(\log N)^{3}},
$$

where $\alpha \in E_{L}, \alpha \notin W_{L}, N=[L: \mathbf{Q}]$, and $C^{\prime}>0$ is a computable absolute constant (inequality (3.4) follows easily from Dobrowolsky's lower bound for heights [D]).

Let the successive minima of \|\| on the lattice $\mathscr{L}\left(E_{L / K}\right)$ be attained at $\varepsilon_{1}, \varepsilon_{2}, \ldots, \varepsilon_{r}$. Thus [GK, pp. 195, 197] the subgroup $E:=\left\langle\varepsilon_{1}, \varepsilon_{2}, \ldots, \varepsilon_{r}\right\rangle$ of $E_{L / K}$ generated by the $\varepsilon_{i}$ has finite index in $E_{L / K}$ and

$$
\begin{gathered}
0<\left\|\varepsilon_{1}\right\| \leq\left\|\varepsilon_{2}\right\| \leq \cdots \leq\left\|\varepsilon_{r}\right\|, \\
\prod_{i=1}^{r}\left\|\varepsilon_{i}\right\| \leq \gamma_{r}^{r / 2} V_{L}\left(E_{L / \tilde{K}}\right),
\end{gathered}
$$

where $\gamma_{r}$ denotes Hermite's constant in dimension $r=r_{L / K}$.

Lemma. Let $\varepsilon_{1}, \varepsilon_{2}, \ldots, \varepsilon_{r}$ be as above and assume that $L / K$ is not unit-weak (see §1). Let $H_{0}=\mathbf{Q}$ and $H_{i}=H_{i-1}(\varepsilon)$. Then there is an integer $T$ such that $H_{T} \neq L, H_{T+1}=L, 0 \leq T<r$, and

$$
\frac{1}{[L: \mathbf{Q}]} \log \left|D_{L}\right| \leq \log ([L: \mathbf{Q}])+\frac{1}{\sqrt{3[L: \mathbf{Q}]}} \sum_{i=1}^{T+1}\left\|\varepsilon_{i}\right\| \sqrt{\left[H_{i}: H_{i-1}\right]^{2}-1},
$$

where $D_{L}$ denotes the absolute discriminant of $L$ and \|\| is given by (3.3). Proof. Proposition 1 implies that there is at least $T<r$ so that $L=$ $\mathbf{Q}\left(\varepsilon_{1}, \varepsilon_{2}, \ldots, \varepsilon_{T+1}\right)$. The inequality then follows from [F, (3.3), (3.14) and Lemma 3.5]. 
Theorem. Let $L / K$ be an extension of number fields and assume that $D_{L}>$ $3 N^{N}$, where $D_{L}$ is the absolute discriminant of $L$ and $N=[L: Q]$. Then

$$
\operatorname{Reg}\left(E_{L / K}\right) \geq \frac{C}{N^{2 r}}\left(\log \left(\frac{\left|D_{L}\right|}{N^{N}}\right)\right)^{m} .
$$

Here $\operatorname{Reg}\left(E_{L / K}\right)$ is the regulator of relative units given by (3.1), $C>0$ is a computable absolute constant, and $r=r_{L}-r_{K}=\operatorname{rank}\left(E_{L / K}\right)$ is the difference of the unit ranks of $L$ and $K$. The nonnegative integer $m$ is positive if $L / K$ is not unit-weak (see §1). In general, $m=m(L / K)=r-\max _{F \subsetneq L}\left\{\operatorname{rank}\left(E_{L / K} \cap F\right)\right\}$, where $F$ runs over all proper subfields of $L$.

The slightly simplified version of the theorem given in $\S 1$ follows from (3.1) and (3.7).

Proof. We first assume that $L / K$ is not unit-weak. From the Lemma and (3.5) we have

$$
\frac{1}{N} \log \left(\frac{\left|D_{L}\right|}{N^{N}}\right) \leq \frac{\left\|\varepsilon_{T+1}\right\|}{\sqrt{3 N}} \sum_{i=1}^{T+1} \sqrt{\left[H_{i}: H_{i-1}\right]^{2}-1} \leq\left\|\varepsilon_{T+1}\right\| \sqrt{\frac{N}{3}},
$$

since $\prod_{i=1}^{T+1}\left[H_{i}: H_{i-1}\right]=N$. From (3.5), (3.6), and (3.4)

$$
\left\|\varepsilon_{T+1}\right\|^{r-T} \leq \prod_{i=T+1}^{r}\left\|\varepsilon_{i}\right\| \leq \frac{\gamma_{r}^{r / 2} V_{L}\left(E_{L / K}\right)}{\left(C^{\prime} / \sqrt{N}(\log N)^{3}\right)^{T}} .
$$

If we put this together with (3.2) and (3.8) and use $\log \left(\left|D_{L}\right| / N^{N}\right)>0$, we find

$$
\begin{aligned}
& \frac{1}{N^{2 r}}\left(\log \left(\frac{\left|D_{L}\right|}{N^{N}}\right)\right)^{r-T} \\
& \quad \leq \frac{\left(([L: K] / 2)^{\left(r_{1}(K)+r_{2}(K)\right) / r} 2^{\left([K: Q]-r_{2}(L)\right) / r} \gamma_{r} / 3 N\right)^{r / 2}}{\left(N C^{\prime} / \sqrt{3}(\log N)^{3}\right)^{T}} \operatorname{Reg}\left(E_{L / K}\right) .
\end{aligned}
$$

If $[L: K] \geq 3$, then $(2.3)$ yields

$$
r=r_{L}-r_{K}=\sum_{\omega \in \infty_{L}}\left(1-\frac{e_{K}(\omega)}{[L: K]}\right) \geq \sum_{\omega \in \infty_{L}} \frac{1}{3} \geq \frac{[L: \mathbf{Q}]}{6} .
$$

Hence, for $[L: K] \geq 2$,

$$
\left(\frac{[L: K]}{2}\right)^{\left(r_{1}(K)+r_{2}(K)\right) / r} \leq\left(\frac{[L: K]}{2}\right)^{6 /[L: K]}<3.003
$$

Note that

$$
[K: \mathbf{Q}]-r_{2}(L) \leq r_{1}(L)+r_{2}(L)-r_{1}(K)-r_{2}(K)=r
$$

and that, for $r>2, \gamma_{r} \leq r / 2.1$. (Proof. Use the inequalities quoted in [CF, (2.9)]). We then have in (3.10)

$$
\left(\left(\frac{[L: K]}{2}\right)^{\left(r_{1}(K)+r_{2}(K)\right) / r} 2^{\left([K: \mathbf{Q}]-r_{2}(L)\right) / r} \frac{\gamma_{r}}{3 N}\right)^{r / 2} \leq 1,
$$


for all $r>0$ (do $e=1$ or 2 separately). Since $T<r<N,(3.10)$ and (3.13) yield

$$
\operatorname{Reg}\left(E_{L / K}\right)>\frac{C}{N^{2 r}}\left(\log \left(\frac{\left|D_{L}\right|}{N^{N}}\right)\right)^{r-T},
$$

with $C>0$ a computable absolute constant. To prove (3.7) we must still show that in (3.14) we can replace $T$ by $\rho:=\max _{F \subsetneq L}\left\{\operatorname{rank}\left(E_{L / K} \cap F\right)\right\}$. Since we assume $D_{L}>3 N^{N}$, it suffices to show $T \leq \rho$. By the lemma, $H_{T}$ is a proper subfield of $L$ containing the $T$ independent relative units $\varepsilon_{1}, \varepsilon_{2}, \ldots, \varepsilon_{T} \in E_{L / K} ;$ hence, $T \leq \rho$. Proposition 1 implies that $m=r-\rho>0$ which concludes the proof when $L / k$ is not unit-weak. from

If $L / K$ is unit-weak then $m=r-\rho=0$ in (3.7). In this case (3.7) follows

Proposition 2. Let $L / K$ be an extension of number fields. Then

$$
\operatorname{Reg}\left(E_{L / K}\right) \geq \frac{c^{r}}{\left(N r(\log N)^{6}\right)^{r / 2}} .
$$

Here $\operatorname{Reg}\left(E_{L / K}\right)$ is the regulator of relative units given by (3.1), $c>0$ is a computable absolute constant, $N=[L: \mathbf{Q}]$, and $r=r_{L}-r_{K}$ is the difference of the unit ranks of $L$ and $K$. (If $r=0,(3.15)$ means the trivial $1 \geq 1$.)

Proof. From (3.4), (3.6), and (3.2) we obtain

$$
\operatorname{Reg}\left(E_{L / K}\right) \geq\left(\frac{C^{2}}{N \gamma_{r}(\log N)^{6}([L: K] / 2)^{\left(r_{1}(K)+r_{2}(K)\right) / r_{2}\left([K: \mathbf{Q}]-r_{2}(L)\right) / r}}\right)^{r / 2} .
$$

Now use (3.11), (3.12), and $\gamma_{r} \leq r$ to obtain (3.15), with $c=C^{\prime} \sqrt{6.006}$.

Corollary. Let $L / K$ (and all notation) be as in the theorem. Suppose further that all proper subfields of $L$ are actually subfields of $K$. Then

$$
\operatorname{Reg}\left(E_{L / K}\right) \geq \frac{1}{N^{2 r}}\left(\log \left(\frac{\left|D_{L}\right|}{N^{N}}\right)\right)^{r} .
$$

Proof. We first dispose of the trivial cases. If $L / K$ is unit-weak, the hypothesis on $K$ implies that case (iii) in Proposition 1 cannot hold. If (ii) holds, so $L$ is CM, then $K$ must be its maximal totally real subfield. Then $r=0$ and (3.16) is trivial. Since case (i) $(L=K)$ is equally trivial, we may assume that $L / K$ is not unit weak. Consider, in the notation of the lemma, $H_{1}=\mathbf{Q}\left(\varepsilon_{1}\right)$. By assumption, either $H_{1} \subseteq K$ or $H_{1}=L$. But $H_{1} \subseteq K$ implies $\varepsilon_{1} \in E_{L / K} \cap K$, which is impossible since $\varepsilon_{1}$ is not a root of unity. Thus, $H_{1}=L$ and so $T=0$ in the lemma. The corollary now follows from (3.13) and (3.10).

The computation of $m=m(L / K)$ in the theorem turns out to be elementary. Let $\mathscr{L}_{L}$ be the logarithmic embedding (2.1). If $M \subset \mathscr{L}_{L}\left(E_{L}\right) \subset \mathbf{R}^{\mathscr{S}_{L}}$ is a lattice, denote its $\mathbf{R}$-span by $\mathbf{R} M$. Thus, $\operatorname{rank}(M)=\operatorname{dim}_{\mathbf{R}}(\mathbf{R} M)$. If $F$ is a subfield of $L$, observe that

$$
\begin{aligned}
& \operatorname{rank}\left(E_{L / K}\right)+\operatorname{rank}\left(E_{F}\right)-\operatorname{rank}\left(E_{L / K} \cap F\right) \\
& \quad=r_{L}-r_{K}+r_{F}-\operatorname{rank}\left(E_{L / K} \cap E_{F}\right) \\
& \quad=\operatorname{rank}\left(E_{L / K} E_{F}\right)=\operatorname{dim} \mathbf{R}\left(\mathbf{R} \mathscr{L}_{L}\left(E_{L / K} E_{F}\right)\right) \\
& \quad=\operatorname{dim} \mathbf{R}\left(\mathbf{R} \mathscr{L}_{L}\left(E_{L / K}\right)+\mathbf{R} \mathscr{L}_{L}\left(E_{F}\right)\right)
\end{aligned}
$$


Dirichlet's unit theorem gives an $\mathbf{R}$-basis of $\mathbf{R} \mathscr{L}_{L}\left(E_{F}\right)$. It also gives one for $\mathbf{R} \mathscr{L}_{L}\left(E_{L / K}\right)$ as the orthogonal complement of $\mathbf{R} \mathscr{L}_{L}\left(E_{K}\right)$ (inside $\mathbf{R} \mathscr{L}_{L}\left(E_{L}\right)$ ). It follows that

$$
m:=\operatorname{rank}\left(E_{L / K}\right)-\max _{F \subsetneq L}\left\{\operatorname{rank}\left(E_{L / K} \cap F\right)\right\}
$$

can be calculated by linear algebra from a knowledge of all the subfields of $L$, without knowing a single unit. To be precise, one has to know, for each subfield $F$ of $L$, the mapping $\mathscr{S}_{L} \rightarrow \mathscr{S}_{F}$ obtained by restricting the embeddings of $L$ to embeddings of $F$.

We conclude with a comment on $\operatorname{Reg}\left(E_{L / K}\right)$ and $\operatorname{Reg}(L) / \operatorname{Reg}(K)$ for $L / K$ unit-weak. We defined in $\S 2$ a subextension $H / k$ and a unit index

$$
u_{L / K}:=\left[E_{L / K}: W_{L} E_{H / k}\right]=1 \text { or } 2 .
$$

On examining the ramification of the archimedean places in $L / K$ and $H / k$ one finds, directly from the definition of $\operatorname{Reg}\left(E_{L / K}\right)$ as a determinant,

$$
\operatorname{Reg}\left(E_{L / K}\right)=2^{r_{H / k}} \operatorname{Reg}\left(E_{H / k}\right) / u_{L / K} .
$$

If we let $L / K$ range over the infinitely many unit-weak extensions associated to the same $H / k$, it is clear from (3.17) that $\operatorname{Reg}\left(E_{L / K}\right)$ assumes at most two values. It follows, mainly from (3.1), that $\operatorname{Reg}(L) / \operatorname{Reg}(K)$ assumes at most $2^{[H: Q]}$ values.

\section{REFERENCES}

[BM1] A.-M. Bergé and J. Martinet, Sur les minorations géométriques des régulateurs, Séminaire de Théorie des Nombres de Paris 1987-1988 (C. Goldstein, ed.), Birkhäuser Verlag, Boston, MA, 1990, pp. 23-50.

[BM2] _-, Notions relatives de régulateur et de hauteur, Acta Arith. 54 (1989), 155-170.

[BM3] _ Minorations de hauteurs et petits régulateurs relatifs, Séminaire de Théorie des Nombres de Bordeaux 1987-1988, Univ. de Bordeaux, 1989.

[CF] A. Costa and E. Friedman, Ratios of regulators in totally real extensions of number fields, J. Number Theory 37 (1991), 288-297.

[D] E. Dobrowolsky, On a question of Lehmer and the number of irreducible factors of a polynomial, Acta Arith. 34 (1979), 391-401.

[F] E. Friedman, Analytic formulas for the regulator of a number field, Invent. Math. 98 (1989), 599-622.

[GK] P. M. Gruber and C. G. Lekkerkerker, Geometry of numbers, North-Holland, Amsterdam, 1987.

[L] E. Landau, Abschätzungen von Charaktersummen, Einheiten und Klassenzahlen, Nachr. Kgl. Ges. Wiss. Göttingen Math.-Phys. Kl. (1918), 79-97; Collected works, vol. 7, Thales Verlag, Essen, 1985, pp. 114-132.

[R1] R. Remak, Über Größenbezienhungen zwischen Diskriminanten und Regulator eines algebraischen Zahlkörpers, Compositio Math. 10 (1952), 245-285.

[R2] _ Über algebraische Zahlkörper mit schwachem Einheitsdefekt, Compositio Math. 12 (1954), 35-80.

[Sie] C.-L. Siegel, Abschätzungen von Einheiten, Nachr. Akad. Wiss. Göttingen Math.-Phys. Kl. 9 (1969), 71-86; Gesammelte Abhandlungen, vol. 4, Springer-Verlag, Berlin, 1979, pp. 66-81. 
[Sil] J. Silverman, An inequality connecting the regulator and discriminant of a number field, J. Number Theory 19 (1984), 437-442.

[Z] R. Zimmert, Ideale kleiner Norm in Idealklassen und eine Regulatorabschätzung, Invent. Math. 62 (1981), 367-380.

Department of Mathematics, American University, Washington, District of Columbia 20016

Current address: Department of Mathematics, George Washington University, Washington, DC 20052

E-mail address: costa@math.gwu.edu

Max Planck Institut für Mathematik, Gottfried Claren Strasse 26, 5300 Bonn 3, GeRMANY 